\title{
Relativistic classical and quantum dynamics in intense crossed laser beams of various polarizations
}

\author{
M. Verschl* and C.H. Keitel ${ }^{\dagger}$ \\ Max-Planck-Institut für Kernphysik, Saupfercheckweg 1, 69117 Heidelberg, Germany \\ Theoretische Quantendynamik, Physikalisches Institut, Albert-Ludwigs-Universität Freiburg, Hermann-Herder-Straße 3, 79104 \\ Freiburg, Germany
}

(Received 24 October 2006; published 2 February 2007)

\begin{abstract}
The dynamics of an electron in crossed laser fields is investigated analytically. Two different standing wave configurations are compared. The counterpropagating laser waves are either linearly or circularly polarized. Both configurations have in common that there are one-dimensional trajectories on which the electron can oscillate with vanishing Lorentz force. The dynamics is analyzed for the situations when the electron moves in the vicinity of these ideal axes. If the laser intensities imply nonrelativistic electron dynamics, the system is described quantum mechanically. A semiclassical treatment renders the strongly relativistic regime accessible as well. To describe relativistic wave packets, the results of the classical analysis are employed for a Monte Carlo ensemble. This allows for a comparison of the wave packet dynamics for both configurations in the strongly relativistic regime. It is found for certain cases that relativity slows down the dynamics, i.e., for higher laser intensities, wave packet spreading and the drift away from the ideal axis of vanishing Lorentz force are shown to be increasingly suppressed.
\end{abstract}

DOI: 10.1103/PhysRevSTAB.10.024001

PACS numbers: 41.75.Jv, 41.75.Ht, 34.80.Kw

\section{INTRODUCTION}

Over the past few decades, experimentally available laser power has increased by several orders of magnitude up to intensities of $10^{22} \mathrm{~W} / \mathrm{cm}^{2}$. The interaction of lasers with atoms involves different regimes, ranging from intensities where the perturbation of atoms by the laser fields is small up to intense laser fields that exceed the fields of the nuclei.

With lasers strong enough such that the tunneling regime is entered, high harmonics of the laser frequency are generated: The superposition of the electric fields of the laser and the nucleus creates a potential barrier where parts of the electron wave packet can tunnel out. As the laser phase reverses, the ionized wave packets return to the core. During this recollision process high harmonic radiation is emitted. For a review of the physics of atoms in intense laser fields see [1-6]. Recollisions and high harmonics generation have a variety of applications such as nonsequential double ionization [7,8], the creation of attosecond pulses $[9,10]$ and coherent ultraviolet light $[11,12]$, probing nuclear motion [13-15], or tomographic imaging of molecular orbits [16].

Recollisions are suppressed if the laser is too intense, because the laser magnetic field exerts a force on the electrons in the laser propagation direction as they are driven by the electric field. Consequently, ionized electrons return to the core at a certain distance (for the electron drift see e.g. [17]). That means the electron velocity has to

\footnotetext{
*Electronic address: mario.verschl@mpi-hd.mpg.de

${ }^{\dagger}$ Electronic address: keitel@mpi-hd.mpg.de
}

remain small compared to the speed of light for effective recollisions.

Today, laser intensities have been reached $[18,19]$ which can accelerate electrons to highly relativistic velocities [20] giving rise to further effects like pair production [20-22], electrostimulated nuclear fission, or nuclear excitation by means of electron impact [23]. However, these intense laser fields do not allow for recollisions because of the above-mentioned electron drift due to the Lorentz force.

Several methods have been proposed to circumvent this problem of the electron drift such as the application of additional electric fields pointing in the laser propagation direction [24], the preacceleration of particles [25,26], to work with antisymmetric molecular states [27], tailoring the laser pulses [28], or the use of positronium where both particles are subject to the same drift [29]. There are further proposals to work with counterpropagating laser waves. With the electric fields of linearly polarized lasers being parallel [30,31], the standing wave has nodes where the magnetic field vanishes and the electric field amplitude is maximal. Electrons move along these nodes where the Lorentz force is suppressed. With circularly polarized counterpropagating laser fields the magnetic field is antiparallel to the electric field, i.e., the Lorentz force vanishes if the electron oscillates in the electric field direction [32]. Crossed field configurations have been discussed in connection with other applications as well, such as particle acceleration [33] or high harmonic generation [34]. Stochastic electron acceleration in two superimposed laser fields has been studied in $[35,36]$.

Experimentally, counterpropagating, strong laser fields have been created by means of a beam splitter and mirrors 
[37]. In this setup, an intensity of the order $10^{19} \mathrm{~W} / \mathrm{cm}^{2}$ was achieved implying relativistic electron dynamics. Further, a new laser system, the Astra Gemini project which is currently under construction, will provide two separate laser beams of intensities up to $10^{22} \mathrm{~W} / \mathrm{cm}^{2}$ (for details see [38]). Thus, crossed laser beams in the highly relativistic regime are expected to be available in the near future.

The purpose of this article is to compare the electron dynamics in counterpropagating laser field configurations of linear and circular polarization and to identify the virtues of the two field configurations. The dynamics is considered in the regions where the Lorentz force is weak and the electron motion is close to a one-dimensional oscillation in the electric field direction. Chaotic behavior does not occur in these regions. The analysis is carried out analytically for free laser-driven electrons which includes classical and quantum mechanical approaches for energies ranging from the nonrelativistic limit to the highly relativistic regime. The analytical solutions allow for intuitive insight into the dynamics of electrons in these standing laser field configurations.

The article has the following structure: In the first section, the two laser configurations, which are to be compared, are introduced. Then the classical equations of motion are analyzed. Solutions are presented both for the nonrelativistic and the strongly relativistic case. In Sec. IV, the problem is treated quantum mechanically, including magnetic field effects of the order $1 / c$. The dynamics of Gaussian wave packets is established. In Sec. V, wave packets are described by means of the classical Monte Carlo method of phase-space averaging. This approach also includes the highly relativistic case.

\section{LASER CONFIGURATIONS}

In the following sections, the dynamics of an electron in a strong laser field will be examined analytically for two different standing wave configurations being introduced now. These configurations are constructed by the superposition of two counterpropagating plane waves which are either linearly or circularly polarized. Both have in common that the Lorentz force due to the magnetic field vanishes on certain axes. However, the difference is that the magnetic field itself is zero only in the first case. This gives rise to a different behavior of electrons moving in the vicinity of these axes.

Throughout the article atomic units (a.u.) will be used, i.e., electromagnetism is described by the Gaussian system with the electron mass, unit charge, and Planck's constant set equal to unity $(e=m=\hbar=1)$.

\section{A. Linearly polarized laser fields}

The laser fields are given by the superposition of two counterpropagating plane waves with equal amplitudes and the same polarization directions. The electromagnetic

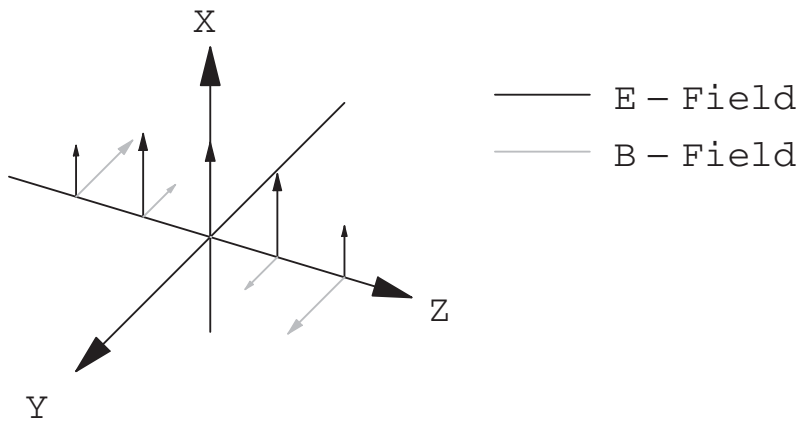

FIG. 1. The field configuration for two counterpropagating laser fields with linear polarization in the $x$ direction is depicted. The laser fields are plane waves propagating in the $z$ direction. The fields are shown in the vicinity of $z=0$, where the magnetic field is small.

fields and the vector potential $\vec{A}$ are given by

$$
\begin{aligned}
\vec{E} & =E_{0} \cos (\omega t) \cos (k z) \hat{x}, \\
\vec{B} & =E_{0} \sin (\omega t) \sin (k z) \hat{y}, \\
\vec{A} & =-E_{0} \frac{c}{\omega} \sin (\omega t) \cos (k z) \hat{x},
\end{aligned}
$$

with the polarization direction $\hat{x}$, and $\hat{z}$ being the laser propagation direction. The explicit form of the two superimposed counterpropagating waves can be recovered by applying the corresponding trigonometric identities.

It is clear that the magnetic field vanishes for $k z=n \pi$ (with $n$ being an integer) whereas the electric field has maximal amplitude. Thus, the dynamics of electrons moving in these planes will be dominated by the electric field. Figure 1 shows the field configuration for the vicinity of $z=0$, i.e., for $(k z)^{2} \ll 1$. The fields do not depend on the coordinates $x$ and $y$.

\section{B. Circularly polarized laser fields}

The other field configuration of interest is the superposition of two counterpropagating, circularly polarized waves. The superimposed fields are given in the following form:

$$
\begin{aligned}
\vec{E} & =E_{0} \cos (\omega t)[\cos (k z) \hat{x}-\sin (k z) \hat{y}], \\
\vec{B} & =-E_{0} \sin (\omega t)[\cos (k z) \hat{x}-\sin (k z) \hat{y}], \\
\vec{A} & =-E_{0} \frac{c}{\omega} \sin (\omega t)[\cos (k z) \hat{x}-\sin (k z) \hat{y}],
\end{aligned}
$$

where the propagation direction is again chosen to be the $z$ direction. Since only one of the two configurations will be considered at a time, the notation for the fields $E, B, A$ is not distinguished. Note here that the magnetic field is antiparallel to the electric field, i.e., if the electrons move in the $E$-field direction, the Lorentz force $\vec{F}=\dot{\vec{x}} / c \times \vec{B}$ vanishes despite the fact that the magnetic field is nonzero (see Fig. 2). 


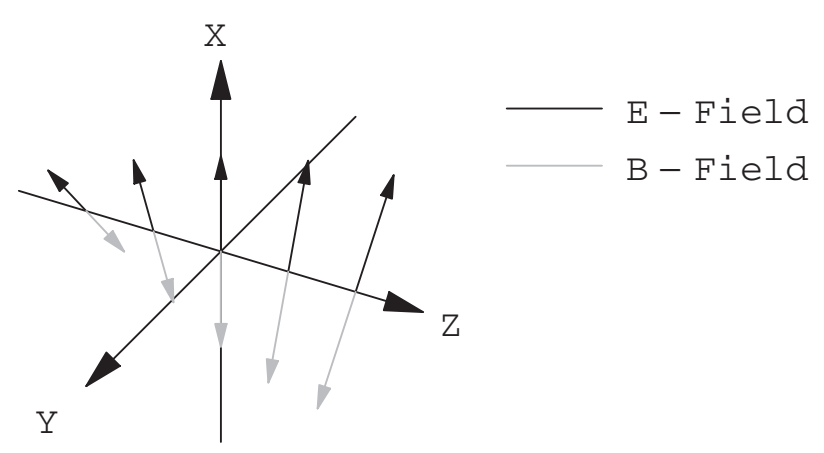

FIG. 2. The field configuration for two counterpropagating laser fields with circular polarization are shown for the vicinity of the plane $z=0$. The plane laser waves propagate in the $z$ direction. In this configuration, the magnetic field is antiparallel to the electric field. The plot is at a time where $\sin \omega t=\cos \omega t$.

\section{RELATIVISTIC CLASSICAL PARTICLE DYNAMICS}

The classical dynamics of an electron moving in the vicinity of an axis with vanishing Lorentz force is examined. The dynamics will be dominated by the simple onedimensional oscillation which is found if the particle is placed exactly on the axis with vanishing initial momentum. Different regimes of laser intensities are considered. Later on, the classical solutions will be employed to describe the dynamics of wave packets by means of a Monte Carlo calculation (see Sec. V).

\section{A. Simplified equations of motion}

A classical particle driven by the laser fields is described by three nonlinear coupled differential equations which are challenging to deal with analytically. However, if the particle dynamics deviates only slightly from the ideal onedimensional motion along an axis, the equations can be simplified, decoupled, and partly solved analytically.

\section{Linear polarization}

The relativistic classical equations of motion are given by

$$
\frac{d}{d t}(\gamma \dot{\vec{x}})=\vec{E}+\frac{\dot{\vec{x}}}{c} \times \vec{B} \quad \text { with } \gamma=\left(1-\frac{\dot{\vec{x}} \cdot \dot{\vec{x}}}{c^{2}}\right)^{-1 / 2},
$$

where the fields are determined by Eqs. (1). To simplify these coupled equations, several approximations can be applied. First, $k z$ is assumed to be small. Consider the following example for near-infrared laser light with the wavelength of $\lambda=800 \mathrm{~nm}$. The absolute value of the wave vector evaluates to $k=2 \pi / \lambda \approx 4.2 \times 10^{-4}$ in atomic units. The particle is expected to move in the vicinity of the ideal plane $z=0$, i.e., as long as the displacement is not greater than a few hundred atomic units, $k z$ remains below a small number of the order
$10^{-1}$. The case of higher displacements means that the electron leaves the ideal axis and the trajectory cannot be considered stable anymore. However, the examples considered here will be in the regime, where $k z$ remains small.

Second, the motion in the $y$ and the $z$ direction is assumed to be nonrelativistic, i.e. $\dot{y} / c$ and $\dot{z} / c$ are small, whereas the motion in the $x$ direction, which can become highly relativistic, is not restricted. This is reasonable, because the initial conditions are nonrelativistic and the acceleration by the electric field is in the $x$ direction, which means that the solution is expected to be only a slight variation of the ideal solution of a one-dimensional oscillation along the $x$ direction. If these assumptions are fulfilled, the small terms can be considered up to linear order and higher order terms are small corrections only. The results of the examples considered later on will comply with these assumptions, i.e., the following approximations are justified:

$$
\begin{aligned}
\sin (k z) & \approx k z, \quad \cos (k z) \approx 1, \\
\left(\frac{\dot{y}}{c}\right)^{2} & \approx\left(\frac{\dot{z}}{c}\right)^{2} \approx \frac{\dot{y}}{c} \cdot \frac{\dot{z}}{c} \approx 0, \\
\frac{\dot{y}}{c} \cdot k z & \approx \frac{\dot{z}}{c} \cdot k z \approx 0 .
\end{aligned}
$$

Now, the simplified set of equations adopts the following form:

$$
\begin{aligned}
\frac{d}{d t}(\gamma \dot{x}) & =E_{0} \cos (\omega t), \\
\frac{d}{d t}(\gamma \dot{y}) & =0, \\
\frac{d}{d t}(\gamma \dot{z}) & =E_{0} \frac{\dot{x}}{c} \sin (\omega t) k z, \\
\gamma & =\left(1-\frac{\dot{x}^{2}}{c^{2}}\right)^{-1 / 2} .
\end{aligned}
$$

From Eqs. (5a) and (5d) the $\gamma$-factor is found to be

$$
\begin{aligned}
\gamma & =\sqrt{1+\left(R \sin (\omega t)+\frac{p_{x}}{c}\right)^{2}} \\
& \approx \sqrt{1+R^{2} \sin ^{2}(\omega t)}+\frac{R \sin (\omega t)}{\sqrt{1+R^{2} \sin ^{2}(\omega t)}} \cdot \frac{p_{x}}{c},
\end{aligned}
$$

with the definition $R \equiv E_{0} /(\omega c)$ and the initial condition for the momentum $\gamma \dot{\vec{x}}(t=0)=\vec{p}_{0} \equiv\left(p_{x}, p_{y}, p_{z}\right)$. For nonrelativistic initial velocities considered here, i.e., for $p_{x} / c \ll 1$, the $\gamma$-factor can be expanded with respect to $p_{x} / c$ and it is seen that the term of first order in $p_{x} / c$ is only a small correction to the leading term.

With the expression for the $\gamma$-factor, the motion in the $x$ direction is given by 


$$
\begin{aligned}
\frac{\dot{x}}{c}= & \frac{R \sin (\omega t)+\frac{p_{x}}{c}}{\sqrt{1+\left[R \sin (\omega t)+\frac{p_{x}}{c}\right]^{2}}} \approx \frac{R \sin (\omega t)}{\sqrt{1+R^{2} \sin ^{2}(\omega t)}} \\
& +\frac{p_{x} / c}{\left[1+R^{2} \sin ^{2}(\omega t)\right]^{3 / 2}}, \\
x \approx & -\frac{c}{\omega} \arcsin \left[\frac{R}{\sqrt{1+R^{2}}} \cos (\omega t)\right] \\
& +p_{x} \int \frac{d t}{\left[1+R^{2} \sin ^{2}(\omega t)\right]^{3 / 2}}+x_{0},
\end{aligned}
$$

where the initial position is defined by $\vec{x}(t=0)=$ $\left(x_{0}, y_{0}, z_{0}\right)$. The approximations are again a first order expansion with respect to $p_{x} / c$.

Finally, the following equations of motion for the $y$ and the $z$ direction are found:

$$
\begin{aligned}
\dot{y} & =\frac{p_{y}}{\sqrt{1+R^{2} \sin ^{2}(\omega t)}} \\
\frac{d}{d t}(\gamma \dot{z}) & =R^{2} \omega^{2} \frac{\sin ^{2}(\omega t)}{\sqrt{1+R^{2} \sin ^{2}(\omega t)}} z
\end{aligned}
$$

where the small terms $p_{x} / c, p_{y} / c$, and $k z$ are again considered to first order only.

Figure 3 shows the motion of an electron for one laser period according to Eqs. (7) and (8). The diagrams depict the position depending on time for the moderately relativistic case of $R=1.5$. It can be seen that the motion is dominated by an oscillatory motion in the electric field direction. The motion in the $z$ direction is unstable, i.e., the velocity in that direction grows rapidly. It is clear that the results become invalid once the approximations in Eq. (4) are violated. However, in this case the electron will have left the vicinity of the $x$ axis, i.e., it will be outside the range of interest if the system is applied to induce recollisions.

Before further investigating Eqs. (8) for certain limits of $R$, the analog procedure will be carried out for the case of circular laser polarization.

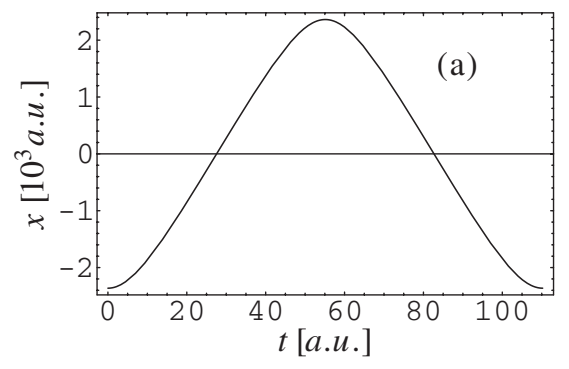

\section{Circular polarization}

The equations of motion (3) for circular laser polarization [see Eqs. (2)] can be simplified by means of the same approximations (4) as applied in the previous section. Then the equations of motion adopt the following form:

$$
\begin{aligned}
\frac{d}{d t}(\gamma \dot{x}) & =E_{0} \cos (\omega t), \\
\frac{d}{d t}(\gamma \dot{y}) & =-E_{0}\left[k z \cos (\omega t)+\frac{\dot{z}}{c} \sin (\omega t)\right], \\
\frac{d}{d t}(\gamma \dot{z}) & =E_{0}\left[\frac{\dot{x}}{c} \sin (\omega t) k z+\frac{\dot{y}}{c} \sin (\omega t)\right], \\
\gamma & =\left(1-\frac{\dot{x}^{2}}{c^{2}}\right)^{-1 / 2} .
\end{aligned}
$$

Comparing these equations with Eqs. (5), it is seen that the dynamics of $\gamma$ and the motion in the $x$ direction are identical to the case of linear laser polarization [see Eqs. (6) and (7)]. Now, Eq. (9b) can be integrated to yield

$$
\dot{y}=\frac{p_{y}-R \omega z \sin (\omega t)}{\sqrt{1+R^{2} \sin ^{2}(\omega t)}} .
$$

With this result, the dynamics in the $z$ direction is found to be

$$
\dot{z} \approx \frac{p_{z}-p_{y}\left\{\arcsin \left[\frac{R}{\sqrt{1+R^{2}}} \cos (\omega t)\right]-\arcsin \frac{R}{\sqrt{1+R^{2}}}\right\}}{\sqrt{1+R^{2} \sin ^{2}(\omega t)}},
$$

where quadratic terms of $p_{x} / c, p_{y} / c$, and $k z$ are dropped again.

The motion of an electron according to Eqs. (7), (10), and (11) with $R=25$ is shown in Fig. 4. The diagram for the motion in the electric field direction becomes an inharmonic triangular shape, because the velocity is limited by the speed of light. As compared to the case of linear polarization (see Fig. 3), there is no instability in the $z$ direction.

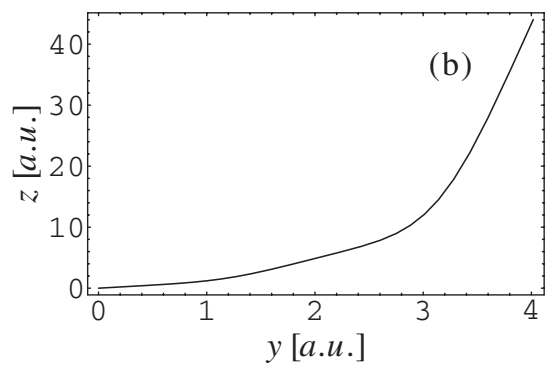

FIG. 3. The motion of an electron in a linearly polarized standing laser field is shown for the moderately relativistic parameter of $R=1.5$. The dynamics is plotted for one complete laser cycle with the initial momentum of the electron $p_{x}=0, p_{y}=p_{z}=0.05$ a.u. Part (a) shows the time-dependent motion in the polarization direction and in (b) the trajectory in the $y$ - $z$ plane is depicted. It is seen that the motion in the $z$ direction is unstable. 

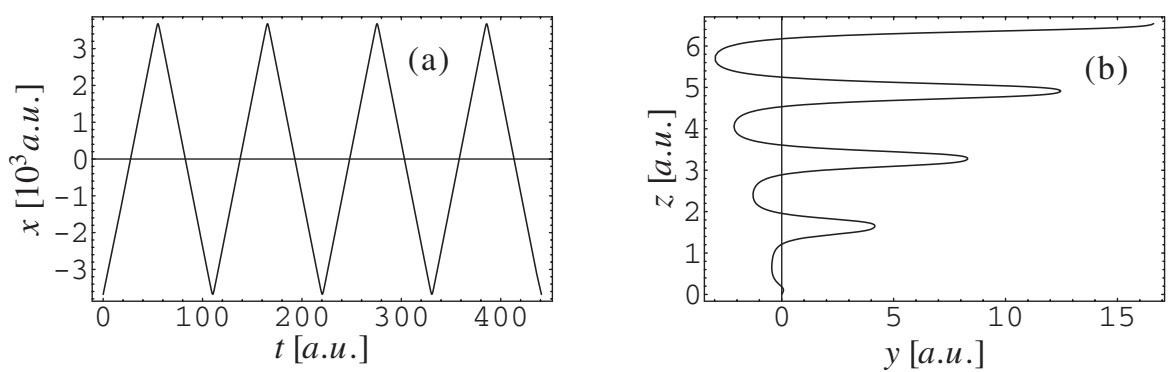

FIG. 4. The motion of an electron in a circularly polarized standing laser field is depicted for four laser cycles. Part (a) shows the dynamics in the $x$ direction whereas (b) shows the trajectory in the $y-z$ plane, i.e., perpendicular to the electric field. With the parameter $R=25$ the motion is relativistic. The initial momentum is chosen to be $p_{x}=0, p_{y}=p_{z}=0.05$ a.u.

\section{B. Nonrelativistic limit}

The equations of motion derived above can be solved completely, if the nonrelativistic limit is considered. In the following, the parameter $R$ is assumed to be small such that $R^{2} \ll 1$. According to Eq. (6), the $\gamma$-factor reduces to one and thus the problem becomes nonrelativistic. Then the equations of motion simplify from the beginning.

\section{Linear polarization}

If terms of the order $R^{2}$ are neglected in the equations of motion (5), the system can be solved immediately. The following nonrelativistic solution is found:

$$
\begin{aligned}
& x=-\frac{R c}{\omega} \cos (\omega t)+p_{x} t+x_{0}, \\
& y=p_{y} t+y_{0}, \\
& z=p_{z} t+y_{0} .
\end{aligned}
$$

The motion in the $y$ and the $z$ direction is equal to that of a free particle. In the polarization direction, one finds a harmonic oscillation caused by the electric field of the laser with an additional drift term $p_{x} t$.

\section{Circular polarization}

In the nonrelativistic limit where $R^{2} \ll 1$, the equations of motion (9) for circular laser polarization can be solved as well. The following result is found:

$$
\begin{aligned}
x= & -\frac{R c}{\omega} \cos (\omega t)+p_{x} t+x_{0}, \\
y= & p_{y} t+y_{0}+R\left(t-\frac{1}{\omega} \sin (\omega t)\right) p_{z} \\
& -R\left(p_{z} t+z_{0}\right)[1-\cos (\omega t)], \\
z= & p_{z} t+z_{0}+R\left(t-\frac{1}{\omega} \sin (\omega t)\right) p_{y} .
\end{aligned}
$$

The dynamics in the $x$ direction is equal to the case of linear polarization [compare Eq. (12a)], whereas the dynamics in the other directions differ slightly by the terms proportional to $R$. This is due to the different magnetic fields of the two configurations.

\section{Highly relativistic case}

The fully relativistic equations of motion can also be simplified for the interesting case that the motion in the $x$ direction becomes highly relativistic. In this limit, the approximation $R^{2} \gg 1$ will be applied, i.e., terms of the order one are neglected with respect to those of the order $R^{2}$. With this approximation, the position can be determined analytically at several interesting instants of time, e.g., after a quarter of a period when the particle has reached its maximum speed or after a full period. According to Eq. (6), the maximum value of the $\gamma$-factor becomes $\gamma_{\max } \approx R$.

\section{Linear polarization}

To integrate the equation of motion for the $y$ direction (8a) two different periods of time are considered:

$$
\begin{aligned}
\dot{y} & =\frac{p_{y}}{\sqrt{1+R^{2} \sin ^{2}(\omega t)}} \\
& =\left\{\begin{array}{ll}
\frac{p_{y}}{\sqrt{1+(R \omega t)^{2}}}, & \sin ^{2}(\omega t) \ll 1 \\
\frac{p_{y}}{R \sin (\omega t)}, & R^{2} \sin ^{2}(\omega t) \gg 1 .
\end{array} .\right.
\end{aligned}
$$

These terms can be integrated to yield

$y=\frac{p_{y}}{R \omega} \operatorname{arsinh}(R \omega t)+y_{0}, \quad$ for $\sin ^{2}(\omega t) \ll 1$,

$y=\frac{p_{y}}{R \omega} \log \left(\tan \frac{\omega t}{2}\right)+\tilde{y_{0}}, \quad$ for $R^{2} \sin ^{2}(\omega t) \gg 1$.

Now, the integration constant $\tilde{y_{0}}$ needs to be matched such that the function is continuous. At the matching point $\tilde{t}$, which needs not to be specified here, both conditions in Eq. (14) $\sin ^{2}(\omega \tilde{t}) \ll 1$ and $R^{2} \sin ^{2}(\omega \tilde{t}) \gg 1$ have to be valid. These are employed for the following approximations:

$$
\begin{aligned}
& \operatorname{arsinh}(R \omega \tilde{t})=\log \left[R \omega \tilde{t}+\sqrt{1+(R \omega \tilde{t})^{2}}\right] \approx \log (2 R \omega \tilde{t}) \\
& \log \left(\tan \frac{\omega \tilde{t}}{2}\right) \approx \log \left(\frac{\omega \tilde{t}}{2}\right) .
\end{aligned}
$$


The condition that (15) be continuous gives

$$
\tilde{y_{0}}=\frac{p_{y}}{R \omega} \log (4 R)+y_{0} .
$$

The error of this result decreases as $R$ increases, because the requirements $R^{2} \sin ^{2}(\omega t) \gg 1$ and $\sin ^{2}(\omega t) \ll 1$ can be better fulfilled simultaneously in the area where the two functions (15a) and (15b) have to match. A comparison of this result for $y(\omega t=\pi / 2)$ with a numerical integration of Eq. (8a) for the moderate value of $R=10$ gives only a relative error of less than $0.2 \%$.

Note, that the distance the electron travels in the $y$ direction is proportional to $R$, i.e., the greater the laser intensity becomes, the closer the electron stays to the initial position $y_{0}$. This can be understood as follows. According to Eq. (5b) the momentum in the $y$ direction is constant: $\gamma \dot{y}=p_{y}$. This means, if the $\gamma$-factor becomes high due to a relativistic motion in the $x$ direction, $\dot{y}$ has to become small.

To investigate the dynamics in the $z$ direction, the differential equation (8b) has to be analyzed. It can be rearranged to the form

$$
\begin{aligned}
{\left[1+R^{2} \sin ^{2}(\omega t)\right] \frac{\ddot{z}}{\omega^{2}}=} & -R^{2} \sin (\omega t) \cos (\omega t) \frac{\dot{z}}{\omega} \\
& +R^{2} \sin ^{2}(\omega t) z .
\end{aligned}
$$

The equation is examined separately for two different time domains and reduces to the following simplified equations:

$$
\begin{aligned}
{\left[1+(R \omega t)^{2}\right] \frac{\ddot{z}}{\omega^{2}} } & =-R^{2} \omega t \frac{\dot{z}}{\omega}+(R \omega t)^{2} z, \quad \text { for } \sin ^{2}(\omega t) \ll 1, \\
\frac{\ddot{z}}{\omega^{2}} & =-\cot (\omega t) \frac{\dot{z}}{\omega}+z, \quad \text { for } R^{2} \sin ^{2}(\omega t) \gg 1 .
\end{aligned}
$$

The first equation can be solved perturbatively:

$$
\begin{gathered}
{\left[R^{-2}+(\omega t)^{2}\right] \frac{\ddot{z}}{\omega^{2}}+\omega t \frac{\dot{z}}{\omega}-(\omega t)^{2} z_{0}=\lambda(\omega t)^{2}\left(z-z_{0}\right),} \\
z(t)=f_{0}(t)+\lambda f_{1}(t)+\cdots .
\end{gathered}
$$

The parameter $\lambda$ is equal to unity and serves only to count the orders of the perturbation expansion. By inserting the ansatz (20b) into Eq. (20a), a differential equation is found for each order of $\lambda$ which can be solved successively. With the right-hand side of Eq. (20a) being a small perturbation, the series converges quickly. The dominating part of the solution will be given by the zeroth order term $f_{0}(t)$, whereas the first order correction can be used to control the error.

The inhomogeneous linear differential equation of zeroth order in $\lambda$ is solved by the ansatz

$$
\frac{\dot{f}_{0}}{\omega}=\frac{p(t)}{\sqrt{1+(R \omega t)^{2}}},
$$

which yields the following result:

$$
\begin{aligned}
& \dot{z}=\frac{p_{z}}{\sqrt{1+(R \omega t)^{2}}}+\frac{\omega}{2}\left(\omega t-\frac{\operatorname{arsinh}(R \omega t)}{R \sqrt{1+(R \omega t)^{2}}}\right) z_{0} \\
& z=\frac{p_{z}}{R \omega} \operatorname{arsinh}(R \omega t)+z_{0}\left(1+\frac{(\omega t)^{2}}{4}-\frac{\operatorname{arsinh}^{2}(R \omega t)}{4 R^{2}}\right) .
\end{aligned}
$$

The error of considering only the lowest order in the expansion (20b) is small, which can be shown by determining an approximation for the first order correction term $f_{1}(t)$. Therefore, $R \gg 1$ can be employed to simplify $f_{0}(t)$ and one can derive the approximate correction term

$$
f_{1}(t)=\frac{1}{64}(\omega t)^{4} z_{0}
$$

which is in fact only a small correction for the times $\sin ^{2}(\omega t) \ll 1$ where the differential equation is valid.

Now, the differential equation has to be solved for the times when $R^{2} \sin ^{2}(\omega t) \gg 1$ [see Eq. (19b)]. Linearity requires the solution to be of the form

$$
\begin{aligned}
& z=h_{1}(\omega t) \frac{\tilde{p}_{z}}{\omega}+h_{2}(\omega t) \tilde{z_{0}}, \\
& \dot{z}=\dot{h_{1}}(\omega t) \frac{\tilde{p}_{z}}{\omega}+\dot{h_{2}}(\omega t) \tilde{z_{0}}=h_{1}^{\prime}(\omega t) \tilde{p}_{z}+h_{2}^{\prime}(\omega t) \omega \tilde{z_{0}},
\end{aligned}
$$

i.e. $z(t)$ can be written as a linear combination of two timedependent functions $h_{1}(t)$ and $h_{2}(t)$, whereas the initial conditions at the initial time $\tilde{t}$ are given by $z(t=\tilde{t})=\tilde{z}_{0}$ and $\dot{z}(t=\tilde{t})=\tilde{p}_{z}$. The prime in Eq. (24b) denotes the derivative with respect to $(\omega t)$. Since the differential equation does not depend on $R$, the coefficients can be calculated numerically for certain times $t$ without loss of generality. The constants $h_{1 / 2}(\omega t)$ and $h_{1 / 2}^{\prime}(\omega t)$ are fixed by calculating the solution for two linearly independent pairs of initial conditions. An interesting instant is when the particle reaches its maximal velocity, i.e., for $\omega t=$ $\pi / 2$. Equations (19a) and (19b) are coupled at the time $\tilde{t}$, which needs to be chosen suitably such that the validity of both limits $\sin ^{2}(\omega t) \ll 1$ and $R^{2} \sin ^{2}(\omega t) \gg 1$ is ensured. With $\omega \tilde{t}=0.1$ good agreement with the original differential equation (18) is expected for values $R>50$, but as it will turn out, the results will be valid for values much 
smaller. The coefficients are found to be

$$
\begin{array}{ll}
h_{1}\left(\omega t=\frac{\pi}{2}\right)=0.44, & h_{2}\left(\omega t=\frac{\pi}{2}\right)=1.79 \\
h_{1}^{\prime}\left(\omega t=\frac{\pi}{2}\right)=0.38, & h_{2}^{\prime}\left(\omega t=\frac{\pi}{2}\right)=1.32 .
\end{array}
$$

The initial velocity $\tilde{p}_{z}$ and the initial position $\tilde{z}_{0}$ are determined by Eqs. (22) for the preceding time domain. The results for $z(\omega t=\pi / 2)$ and $\dot{z}(\omega t=\pi / 2)$ can be written in matrix form:

$$
\left(\begin{array}{c}
z\left(\frac{\pi}{2}\right) \\
\dot{z}\left(\frac{\pi}{2}\right) / \omega
\end{array}\right)=B\left(\begin{array}{c}
z(\tilde{t}) \\
\dot{z}(\tilde{t}) / \omega
\end{array}\right)=B \cdot A\left(\begin{array}{c}
z_{0} \\
p_{z} / \omega
\end{array}\right)
$$

with the definitions

$$
\begin{aligned}
A & =\left(\begin{array}{cc}
1-\frac{1}{4 R^{2}} \log \left(\frac{R}{5}\right)^{2} & \frac{1}{R} \log \left(\frac{R}{5}\right) \\
0.05-\frac{5 \log \left(\frac{R}{5}\right)}{R^{2}} & \frac{10}{R}
\end{array}\right), \\
B & =\left(\begin{array}{ll}
1.79 & 0.44 \\
1.32 & 0.38
\end{array}\right) .
\end{aligned}
$$

Matrix $A$ follows from Eq. (22), which can be simplified by means of the same approximations already applied to decompose the original differential equation [see Eqs. (18) and (19)], i.e. $\sin ^{2}(\omega \tilde{t}) \approx(\omega \tilde{t})^{2} \ll 1$ and $R^{2} \sin ^{2}(\omega \tilde{t}) \approx R^{2}(\omega \tilde{t})^{2} \gg 1$. This also implies the approximation (16a). $B$ is determined by Eqs. (24) and (25).

In the same way, the position and the velocity can be calculated after half a period:

$$
\left(\begin{array}{c}
z(\pi) \\
\dot{z}(\pi) / \omega
\end{array}\right)=D \cdot C\left(\begin{array}{c}
z\left(\frac{\pi}{2}\right) \\
\dot{z}\left(\frac{\pi}{2}\right) / \omega
\end{array}\right)=D \cdot C \cdot B \cdot A\left(\begin{array}{c}
z_{0} \\
p_{z} / \omega
\end{array}\right)
$$

The matrix $C$ is again calculated numerically by solving the differential equation (19b) in the interval $\pi / 2<\omega t<$ $\pi-\omega \tilde{t}$. $D$ can be constructed from Eq. (19a), because the differential equation (18) looks the same for the intervals $(\pi-\omega \tilde{t})<\omega t<\pi$ and $0<\omega t<\omega \tilde{t}$ (a time shift of half a period leaves the equation invariant), i.e.,

$$
\begin{aligned}
\left(\begin{array}{c}
z(\pi) \\
\dot{z}(\pi) / \omega
\end{array}\right) & =D\left(\begin{array}{c}
z(\pi-\omega \tilde{t}) \\
\dot{z}(\pi-\omega \tilde{t}) / \omega
\end{array}\right) \\
& \Leftrightarrow D^{-1}\left(\begin{array}{c}
z(0) \\
\dot{z}(0) / \omega
\end{array}\right)=\left(\begin{array}{c}
z(-\omega \tilde{t}) \\
\dot{z}(-\omega \tilde{t}) / \omega
\end{array}\right)
\end{aligned}
$$

where $D^{-1}$ follows immediately from Eqs. (22) with the time given by $t=-\tilde{t}$. Then the following results are found:

$$
C=\left(\begin{array}{cc}
3.81 & 4.41 \\
13.20 & 17.89
\end{array}\right), \quad D=\left(\begin{array}{ll}
1 & \frac{\log (R / 5)}{10} \\
\frac{1}{20} R & \frac{10}{R}
\end{array}\right)
$$

To obtain $D$, a term $\log (R / 5) / 200=\log (2 R \omega \tilde{t}) \omega \tilde{t} / 2$ has been considered small as compared to one. In principle, this term could become large, but for the corresponding high values of $R$ the matching point $\tilde{t}$ can be chosen to be much smaller which increases the precision and changes the matrix entries.

The coefficients in the differential equation (18) are periodic in $\omega t=\pi$, i.e. $z$ and $\dot{z}$ can be determined at any time $\omega t=n \cdot \pi / 2$ ( $n$ being an integer) by subsequently multiplying the matrices $B \cdot A$ and $D \cdot C$.

There is an important difference between the dynamics in the $y$ and the $z$ direction. Whereas the motion in the $y$ direction freezes as $R$ becomes high, the motion in the $z$ direction is unstable. By multiplying the corresponding matrices, the following limits for high values of $R$ are found:

$$
\begin{aligned}
z\left(\frac{\pi}{2}\right) & =1.81 z_{0}, \\
z(\pi) & \approx(5.11+4.78 \log R) z_{0} .
\end{aligned}
$$

Thus, the distance to the plane $z=0$ roughly doubles during a quarter of a period and depending on $R$ it increases further for half a period.

For $R>30$ the entries of the matrices $B \cdot A$ and $D \cdot C$. $B \cdot A$ deviate from the exact results by less than about $1 \%$. The error increases for smaller values, but for $R=10$ it is still of the order of 5\% only. The essential result is that in the strongly relativistic regime the motion in the $z$ direction becomes unstable for times greater than half a laser period. This instability originates from the nonvanishing magnetic field in the vicinity of the plane $z=0$. Obviously, the magnetic field in the $y$ direction is directed such that, together with the motion in the $x$ direction, the Lorentz force pushes the particle farther away from the plane $z=0$.

\section{Circular polarization}

Now, the corresponding analysis will be carried out for circular laser polarization. The location and the velocity of the electron will be calculated for $\omega t=\pi / 2$ such that a direct comparison with the case of linear polarization will be at hand. A further instant of interest is the time when the equations of motion adopt their original form again, which is after a full laser period. Knowing the state of the particle after a period, this allows for an analysis of the long-term behavior.

In the strongly relativistic case, i.e., for $R^{2} \gg 1$ Eq. (11) can be simplified by means of 


$$
\begin{aligned}
\frac{R}{\sqrt{1+R^{2}}} & \approx 1, \\
\arcsin [\cos (\omega t)] & = \begin{cases}\frac{\pi}{2}-\omega t, & 0 \leq \omega t<\pi \\
\omega t-\frac{3 \pi}{2}, & \pi \leq \omega t<2 \pi .\end{cases}
\end{aligned}
$$

Then one finds

$$
\begin{aligned}
\dot{z}= & \frac{1}{\sqrt{1+R^{2} \sin ^{2}(\omega t)}} \\
& \times \begin{cases}\left(p_{z}+p_{y} \omega t\right), & 0 \leq \omega t<\pi \\
{\left[p_{z}+p_{y}(2 \pi-\omega t)\right],} & \pi \leq \omega t<2 \pi .\end{cases}
\end{aligned}
$$

Considering that $\quad \int_{0}^{\pi}(\omega t-\pi / 2) /[1+$ $\left.R^{2} \sin ^{2}(\omega t)\right]^{-1 / 2} d(\omega t)=0$, the integration of Eq. (33) over a whole period yields

$$
\begin{aligned}
z(2 \pi) & =\int_{0}^{2 \pi} \frac{\left(p_{z}+\frac{\pi}{2} p_{y}\right) d(\omega t)}{\omega \sqrt{1+R^{2} \sin ^{2}(\omega t)}} \\
& =4\left(p_{z}+\frac{\pi}{2} p_{y}\right) \frac{\log (4 R)}{R \omega}+z_{0} .
\end{aligned}
$$

The integral in Eq. (34) has already been solved in the previous section [compare Eq. (14)].

If the integration is only for a quarter of a period, the following integral is split up into regions where either $\sin ^{2}(\omega t) \approx(\omega t)^{2} \ll 1$ or $R^{2} \sin ^{2}(\omega t) \gg 1$ can be exploited; $\epsilon$ is a matching point where both conditions are valid:

$$
\int_{0}^{\pi / 2} d(\omega t) \frac{\omega t}{\sqrt{1+R^{2} \sin ^{2}(\omega t)}} \approx \int_{0}^{\epsilon} d(\omega t) \frac{\omega t}{\sqrt{1+(R \omega t)^{2}}}+\frac{1}{R} \int_{0}^{\pi / 2} d(\omega t) \frac{\omega t}{\sin (\omega t)}-\frac{1}{R} \int_{0}^{\epsilon} d(\omega t) \frac{\omega t}{\sin (\omega t)} \approx \frac{1.832}{R}-\frac{1}{R^{2}}
$$

The first integral is carried out analytically, the second one can be evaluated numerically without spoiling the dependence on $R$ and the third one simplifies because $(\omega t)^{2} \ll 1$ for $t \leq \epsilon$. Finally, employing both conditions at the matching point $R^{2} \sin ^{2}(\epsilon) \approx(R \epsilon)^{2} \gg 1$, the result becomes independent of $\epsilon$.

In all, the $z$ position after a quarter of a period is given by

$$
z\left(\frac{\pi}{2}\right)=p_{z} \frac{\log (4 R)}{R \omega}+\frac{p_{y}}{R \omega}\left(1.832-\frac{1}{R}\right)+z_{0}
$$

The position in the $y$ direction is obtained by integrating Eq. (10). Therefore, the following integral needs to be analyzed:

$$
-\int_{0}^{\omega t} z \frac{R \cdot \sin (\omega t) d(\omega t)}{\sqrt{1+R^{2} \sin ^{2}(\omega t)}}=z \arcsin [\cos (\omega t)]-\frac{\pi}{2} z_{0}-\int_{0}^{\omega t} z^{\prime} \arcsin [\cos (\omega t)] d(\omega t)
$$

This is obtained by a partial integration and by employing Eq. (32a).

For a whole period, i.e., for $\omega t=2 \pi$, and by employing Eq. (32b), the following integral occurs which can be solved with the same reasoning as applied to Eq. (35):

$$
\begin{aligned}
& \int_{0}^{\pi \frac{\pi^{2}}{4}-\omega t(\pi-\omega t)} \frac{\frac{\pi^{2}}{4}}{\sqrt{1+R^{2} \sin ^{2}(\omega t)}} d(\omega t) \approx \int_{0}^{\pi} \frac{\int_{0}^{2}}{\sqrt{1+R^{2} \sin ^{2}(\omega t)}} d(\omega t)-2 \frac{\omega t(\pi-\omega t)}{\sqrt{1+(R \omega t)^{2}}} d(\omega t) \\
& -\frac{2}{R}\left[\int_{0}^{\pi / 2} \frac{\omega t(\pi-\omega t)}{\sin (\omega t)} d(\omega t)-\int_{0}^{\epsilon} \frac{\omega t(\pi-\omega t)}{\sin (\omega t)} d(\omega t)\right] \approx \frac{\pi^{2}}{2} \frac{\log 4 R}{R}-\frac{8.414}{R}+\frac{2 \pi}{R^{2}} .
\end{aligned}
$$

The first integral has the same form as in Eq. (34). A term $\left(2 R^{3}\right)^{-1} \operatorname{arsinh}(R \epsilon)$ has been dropped here, because the dependence on $R^{-3}$ makes it only a minor correction. Summarizing, the $y$ position after a whole period is found to be

$$
y(2 \pi)=\frac{4 \log 4 R}{R \omega}\left[\left(1+\frac{\pi^{2}}{2}\right) p_{y}+\frac{\pi}{2} p_{z}\right]-\frac{p_{y}}{R \omega}\left(16.83-\frac{2 \pi}{R}\right)+y_{0} .
$$


Considering the $y$ position after a quarter of a period, integrals occur which have already been considered [see Eqs. (35) and (38)] and the following result is found:

$$
\begin{aligned}
y(\pi / 2)= & \frac{\log 4 R}{R \omega}\left(p_{y}-\frac{\pi}{2} p_{z}\right)-\frac{p_{y}}{R \omega}\left(7.085-\frac{\pi}{2 R}\right) \\
& +\frac{p_{z}}{R \omega}\left(1.832-\frac{1}{R}\right)-\frac{\pi}{2} z_{0}+y_{0} .
\end{aligned}
$$

Considering the limit of high values of $R$, this expression reduces to

$$
y(\pi / 2)=-\frac{\pi}{2} z_{0}+y_{0} .
$$

Here, the initial $z$ position appears with a factor similar to the case of linear polarization [compare Eq. (31a)]. However, after a full period the particle returns to its initial position $\left(y_{0}, z_{0}\right)$ according to Eqs. (34) and (39). This means that the configuration of circularly polarized light becomes superior to the other in the long-term behavior. This can be further investigated. The particle state after a full period is given by $\dot{y}=p_{y}, \dot{z}=p_{z}$, and Eqs. (34) and (39). The equations of motion are periodic, i.e., these results can be used to obtain the particle state after any complete period by consecutively calculating the initial conditions of the next period. The following result is found for the state after $n$ full periods:

$$
\begin{gathered}
\dot{y}(\omega t=2 \pi n)=p_{y}, \quad \dot{z}(\omega t=2 \pi n)=p_{z}, \\
y(\omega t=2 \pi n)=n\left[y(\omega t=2 \pi)-y_{0}\right]+y_{0}, \\
z(\omega t=2 \pi n)=n\left[z(\omega t=2 \pi)-z_{0}\right]+z_{0} .
\end{gathered}
$$

After any full period, the momentum takes its initial value whereas the distance to the origin increases linearly, i.e., there is no exponentially growing instability as in the case of linear polarization. The distance from the $x$ axis even grows more slowly as the laser intensity increases which is seen from the $R^{-1}$-dependence of $y(\omega t=2 \pi)$ and $z(\omega t=$ $2 \pi)$ [see Eqs. (34) and (39)].

\section{QUANTUM MECHANICAL TREATMENT}

In the following, a quantum mechanical description of the electron dynamics will be developed. As opposed to classical mechanics, the wave character of the particle is taken into account. With the quantum mechanical results at hand it will be seen how they are related to the classical description.

\section{A. Solution of the Schrödinger equation}

The quantum mechanical dynamics of an electron in a laser field is determined by the Schrödinger equation:

$$
\begin{aligned}
i \frac{\partial}{\partial t} \psi & =\hat{H} \psi=\frac{1}{2}\left[-i \vec{\nabla}-\frac{1}{c} \vec{A}\right]^{2} \psi \\
& =\frac{1}{2}\left[-\vec{\nabla}^{2}+\frac{2 i}{c} \vec{A} \cdot \vec{\nabla}+\frac{1}{c^{2}} A^{2}\right] \psi
\end{aligned}
$$

The vector potential is assumed to be given in the Coulomb gauge $(\vec{\nabla} \cdot \vec{A}=0)$. For the two laser configurations defined by the vector potentials (1c) and (2c), this equation can be solved for a spatially restricted area. It will be assumed that the particle is found close to the plane $z=0$, i.e., the calculation will be carried out to linear order in $(k z)$. Once the solutions are known, wave packets describing localized particles can be constructed which conform to this assumption.

\section{Linear polarization}

The vector potential for the case of linear laser polarization [see Eq. (1c)] reads

$$
\vec{A}=-E_{0} \frac{c}{\omega} \sin (\omega t) \hat{x},
$$

if terms of quadratic or higher order in $(\mathrm{kz})$ are dropped. Then the following ansatz is inserted into the Schrödinger equation (43):

$$
\psi=\exp i\left[\vec{p}_{0} \cdot\left(\vec{x}-\vec{x}_{0}\right)+w(t)\right]
$$

where $\vec{p}_{0}=\left(p_{x}, p_{y}, p_{z}\right)$ denotes the initial electron momentum. This yields a condition for the time-dependent function $w(t)$ which can easily be integrated. Then the solution is found to be

$$
\begin{aligned}
\psi= & \exp i\left[\vec{p}_{0} \cdot\left(\vec{x}-\vec{x}_{0}\right)-\frac{1}{2} \vec{p}_{0}{ }^{2} t+\frac{E_{0}}{\omega^{2}} p_{x} \cos (\omega t)\right. \\
& \left.-\frac{E_{0}^{2}}{4 \omega^{2}}\left(t-\frac{1}{2 \omega} \sin (2 \omega t)\right)\right] .
\end{aligned}
$$

Later on, this result will be used to construct a localized wave packet. But first, the corresponding calculation will be carried out for the case of circular polarization.

\section{Circular polarization}

The simplification that only linear terms of $(k z)$ are considered reduces the vector potential (2c) to the following form:

$$
\vec{A}=-E_{0} \frac{c}{\omega} \sin (\omega t)[\hat{x}-(k z) \hat{y}] .
$$

As opposed to the case of linear laser polarization, the vector potential does not become independent of the $z$ coordinate. The corresponding Hamiltonian reads

$$
\begin{aligned}
\hat{H}= & \frac{1}{2}\left[-\nabla^{2}-2 i \frac{E_{0}}{\omega} \sin (\omega t)\left(\frac{\partial}{\partial x}-(k z) \frac{\partial}{\partial y}\right)\right. \\
& \left.+\frac{E_{0}^{2}}{\omega^{2}} \sin ^{2}(\omega t)\right] .
\end{aligned}
$$


The solution to the Schrödinger equation is found the same way as in the previous case. Because of the dependence on $(k z)$, the ansatz (45) needs to be extended by an additional term $g(t) \cdot k z$ in the exponent:

$$
\psi=\operatorname{expi}\left[\vec{p}_{0} \cdot\left(\vec{x}-\vec{x}_{0}\right)+w(t)+g(t) \cdot k z\right]
$$

By inserting this ansatz into the Schrödinger equation (43), two conditions for the unknown functions $w(t)$ and $g(t)$ are found, one for each order of $(k z)$. Terms of quadratic order in $R=E / \omega c$ can be neglected with respect to unity, because $R$ is a small number in the nonrelativistic regime considered here. Then the wave function $\psi$ is found to be

$$
\begin{aligned}
\psi= & \exp i\left[\vec{p}_{0} \cdot\left(\vec{x}-\vec{x}_{0}\right)-\frac{1}{2} \vec{p}_{0}{ }^{2} t+\frac{E_{0}}{\omega^{2}} p_{x} \cos (\omega t)\right. \\
& -\frac{E_{0}^{2}}{4 \omega^{2}}\left(t-\frac{1}{2 \omega} \sin (2 \omega t)\right)-R p_{y} p_{z}\left(t-\frac{1}{\omega} \sin (\omega t)\right) \\
& \left.+R p_{y}(1-\cos (\omega t)) z\right]
\end{aligned}
$$

With the results of the following section, this solution will be employed to construct a Gaussian wave packet.

\section{B. Gaussian wave packets}

Gaussian wave packets have the convenient property that they are easy to handle and that the widths in both momentum and coordinate space are well defined.

The goal is to superimpose general functions of the form

$$
\phi=\frac{1}{\sqrt{2 \pi}} \exp [i f(x, p, t)]
$$

with the following, normalized Gaussian shaped weighting factor:

$$
\xi=\frac{1}{\sqrt{\Delta p \sqrt{\pi}}} \exp -\frac{\left(p-p_{0}\right)^{2}}{2 \Delta p^{2}}
$$

Here, $f(x, p, t)$ is a general real function which depends on time, one spacial variable $x$, and the corresponding momentum $p$. The maximum of the momentum distribution is given by $p_{0}$ with the width $\Delta p$, where the width is defined such that the probability distribution of momentum $|\xi|^{2}$ drops by a factor of the Euler number with respect to the maximum for the momentum $p_{0} \pm \Delta p$. If the $p$-dependence of $f(x, p, t)$ is only linear or quadratic, the superimposed wave function $\Psi(x)$ and the probability density $|\Psi(x)|^{2}$ have an explicit solution (for a detailed calculation see [17]):

$$
\begin{aligned}
\Psi(x)= & \pi^{-1 / 4}\left[\Delta p^{-1}-i \Delta p f^{\prime \prime}\left(p_{0}\right)\right]^{-1 / 2} \\
& \times \exp \left[i f\left(p_{0}\right)\right] \exp -\frac{\Delta p^{2} f^{\prime}\left(p_{0}\right)^{2}}{1-i \Delta p^{2} f^{\prime \prime}\left(p_{0}\right)} \\
|\Psi(x)|^{2}= & \pi^{-1 / 2}\left[\Delta p^{-2}+\Delta p^{2} f^{\prime \prime}\left(p_{0}\right)^{2}\right]^{-1 / 2} \\
& \times \exp -\frac{f^{\prime}\left(p_{0}\right)^{2}}{\Delta p^{-2}+\Delta p^{2} f^{\prime \prime}\left(p_{0}\right)^{2}}
\end{aligned}
$$

The primes denote derivatives with respect to $p$. The maximum of the wave packet is determined by the first derivative of $f(x, p, t)$, the width by the second.

\section{Quantum mechanical wave packet dynamics}

The results of the last section, together with the solutions of the Schrödinger equation, can now be employed to construct localized wave packets. This means the assumption that the particle is found close to the plane $z=0$, which was used to solve the Schrödinger equation, will finally be met such that consistent solutions will be at hand.

\section{Linear polarization}

The solution (46) of the Schrödinger equation can be written as a product of three factors, each depending only on one momentum variable $p_{x}, p_{y}$, or $p_{z}$. Then Eq. (53b) is applied to each of the factors to obtain the following result for the probability density:

$$
\begin{gathered}
|\psi(x, y, z, t)|^{2}=\pi^{-3 / 2}\left[\Delta p^{-2}+\Delta p^{2} t^{2}\right]^{-3 / 2} \\
\times \exp -\frac{f_{x}^{2}+f_{y}^{2}+f_{z}^{2}}{\Delta p^{-2}+\Delta p^{2} t^{2}} \\
f_{x} \equiv x-x_{0}+\frac{R c}{\omega} \cos (\omega t)-p_{x} t \\
f_{y} \equiv y-y_{0}-p_{y} t, \quad f_{z} \equiv z-z_{0}-p_{z} t .
\end{gathered}
$$

The dynamics of the maximum of this wave packet is found by equating the functions $f_{x, y, z}$ to zero. It is seen that the maximum describes the same trajectories as a classical particle [compare Eqs. (12)].

The wave packet remains spherically symmetric, with the spacial widths $\Delta x^{2}=\Delta p^{-2}+\Delta p^{2} t^{2}$. The spreading dynamics is identical to the one of a free particle (compare for example [39]), i.e., in the configuration of linear laser polarization, wave packet spreading is not affected by the laser fields.

\section{Circular polarization}

The solution of the Schrödinger equation (50) contains a mixed term $p_{y} p_{z}$, i.e., it cannot simply be written as a product of three exponentials where each depends only on one momentum variable. Thus the three-dimensional problem does not reduce to three one-dimensional ones from the beginning. However, a separation can be accomplished 
by means of a coordinate transformation, i.e., the exponent of Eq. (50) is a quadratic form which needs to be diagonalized. The diagonalization is achieved via a rotation about the $x$ axis by an angle of $\pi / 4$. The new coordinates $\tilde{y}$ and $\tilde{z}$ are related to the old ones by the following transformation law:

$$
\begin{array}{ll}
\tilde{y}=\frac{1}{\sqrt{2}}(y+z), & y=\frac{1}{\sqrt{2}}(\tilde{y}-\tilde{z}), \\
\tilde{z}=\frac{1}{\sqrt{2}}(z-y), & z=\frac{1}{\sqrt{2}}(\tilde{y}+\tilde{z}) .
\end{array}
$$

This transformation is applied to both the spacial and the momentum variables $p_{y}$ and $p_{z}$. Then the wave function (50) can be separated according to $\psi\left(p_{x}, \tilde{p}_{y}, \tilde{p}_{z}\right)=$ $\exp \left(p_{x}\right) \cdot \exp \left(\tilde{p}_{y}\right) \cdot \exp \left(\tilde{p}_{z}\right)$. Now, Eq. (53b) is applied to each factor and the probability density turns out to be

$$
\begin{aligned}
|\psi(x, y, z, t)|^{2}= & \pi^{-3 / 2}\left(\Delta w_{x} \Delta w_{+} \Delta w_{-}\right)^{-1} \\
& \times \exp \left[-\frac{f_{x}^{2}}{\Delta w_{x}^{2}}-\frac{f_{y}^{2}}{\Delta w_{+}^{2}}-\frac{f_{z}^{2}}{\Delta w_{-}^{2}}\right] \\
f_{x} \equiv & -\left(x-x_{0}\right)-\frac{R c}{\omega} \cos (\omega t)+p_{x} t, \\
f_{y} \equiv & -\left(\tilde{y}-\tilde{y}_{0}\right)-\frac{1}{2} R[1-\cos (\omega t)](\tilde{y}+\tilde{z}) \\
& +\tilde{p}_{y} t+R \tilde{p}_{y}\left(t-\frac{1}{\omega} \sin (\omega t)\right) \\
f_{z} \equiv & -\left(\tilde{z}-\tilde{z}_{0}\right)+\frac{1}{2} R(1-\cos (\omega t))(\tilde{y}+\tilde{z}) \\
& +\tilde{p}_{z} t-R \tilde{p}_{z}\left(t-\frac{1}{\omega} \sin (\omega t)\right), \\
w_{x} \equiv & \sqrt{\Delta p^{-2}+\Delta p^{2} t^{2}}, \\
\Delta w_{ \pm} \equiv & \sqrt{\Delta p^{-2}+\Delta p^{2}\left[t-R\left(t-\frac{1}{\omega} \sin (\omega t)\right)\right]^{2}} .
\end{aligned}
$$

By equating the arguments of the exponential functions to zero, the motion of the maximum of this Gaussian is found. If the result is transformed back to the original coordinates by means of Eqs. (55) the classical equations of motion (13) are recovered.

The exponents in Eq. (56) contain mixed terms $\tilde{y} \tilde{z}$, i.e., the widths of the wave packet cannot simply be read off. Instead of carrying out a cumbersome coordinate transformation which diagonalizes the quadratic form in the exponent, the widths are examined only after full periods. At these times the mixed terms do not appear.
The following widths after $n$ laser cycles are found:

$$
\begin{aligned}
\Delta x & =\sqrt{\Delta p^{-2}+\Delta p^{2}\left(\frac{\pi n}{\omega}\right)^{2}}, \\
\Delta w_{\tilde{y}, \tilde{z}} & =\sqrt{\Delta p^{-2}+\Delta p^{2}\left(\frac{\pi n}{\omega}(1 \pm R)\right)^{2}} .
\end{aligned}
$$

For full laser cycles, the wave packet widths are similar to those of the linear polarization configuration [compare Eq. (54)]. Differences are given by the factors $(1 \pm R)$ which only slightly deviate from unity in the nonrelativistic regime considered in this section. The deviations originate from the nonvanishing magnetic field of this laser configuration.

\section{RELATIVISTIC WAVE PACKET APPROACH}

For a fully relativistic quantum mechanical description of wave packets, one has to deal with relativistic wave equations. One approach is the numerical propagation of wave packets on a grid by means of the Dirac equation [40-46]. However, these calculations remain challenging due to limited computing power. An analytical solution of the Dirac equation for an electron in a propagating laser field is given in [47]. A further method to describe the electron wave packet dynamics is phase-space averaging where an ensemble of classical trajectories is used to mimic the wave packet. The initial conditions of the electron are not known exactly because the electron is subject to some quantum mechanical initial state which has to conform with Heisenberg's uncertainty principle. The probability to find the electron in a certain volume is then determined by varying the initial conditions and checking how many classical trajectories end in this volume. This classical Monte Carlo description has been applied to describe both nonrelativistic [48-50] and relativistic $[32,51,52]$ laser-atom processes. Here, an analytical implementation of phase-space averaging is chosen which makes use of the classical solutions derived in Sec. III. This approach describes wave packet spreading but it does not include intrinsic quantum mechanical effects like interferences or spin. However, in this one-particle scheme interference effects are expected to play a minor role and spin effects can be neglected for laser intensities that are of interest here [6,53].

For calculating reaction rates such as for electron-core collisions, the spreading dynamics of wave packets is essential. In general, the more wave packets spread in the directions perpendicular to their directions of motion, the more the reaction rates decrease. Therefore, the electron probability densities for the two configurations will be determined for the $y-z$ plane.

\section{A. Circular polarization}

The solutions of the classical equations of motion are given in a form where the dependence of the initial con- 
ditions is linear. For the motion in the $y$ and the $z$ direction, they have the following structure:

$$
\begin{aligned}
& y(t)=g_{1}(t) \cdot p_{y}+g_{3}(t) \cdot p_{z}+g_{5}(t) \cdot z_{0}+y_{0}, \\
& z(t)=g_{2}(t) \cdot p_{z}+g_{4}(t) \cdot p_{y}+z_{0} .
\end{aligned}
$$

These equations need to be inverted, such that the momentum is found which is needed for a classical particle to travel from the initial position $\left(y_{0}, z_{0}\right)$ to some other point $(y, z)$ at time $t$. Then the probability to find the particle there is proportional to the probability $w\left(p_{y}, p_{z}\right)$ that the particle has the required initial momentum $\left(p_{y}, p_{z}\right)$. The initial state $\tilde{\psi}_{0}$ is given by the product of two Gaussians as in Eq. (52) which give the following distribution for the initial momenta:

$$
w\left(p_{y}, p_{z}\right) \propto \exp \left[-\frac{\left(p_{y}-p_{y_{m}}\right)^{2}+\left(p_{z}-p_{z_{m}}\right)^{2}}{\Delta p^{2}}\right] .
$$

$\left(p_{y_{m}}, p_{z_{m}}\right)$ is the maximum of the initial momentum distribution. Now, the initial position $\left(y_{0}, z_{0}\right)$ is distributed according to the probability density following from the initial state in coordinate space $\psi_{0}$, which is related to $\tilde{\psi}_{0}$ by a Fourier transformation. Thus, the probability density is given by

$$
\begin{aligned}
\rho(y, z, t) \propto & \iint d y_{0} d z_{0} w\left(p_{y}, p_{z}\right) \cdot\left|\psi_{0}\left(y_{0}, z_{0}\right)\right|^{2} \\
= & \iint d y_{0} d z_{0} \exp -\left[\frac{\left(p_{y}-p_{y_{m}}\right)^{2}+\left(p_{z}-p_{z_{m}}\right)^{2}}{\Delta p^{2}}\right. \\
& \left.+\Delta p^{2}\left(y_{0}-y_{m}\right)^{2}+\Delta p^{2}\left(z_{0}-z_{m}\right)^{2}\right]
\end{aligned}
$$

where $\left(y_{m}, z_{m}\right)$ is the initial position of the wave packet maximum. This double integral can be carried out in principle, but it is much more instructive to consider the interesting special case of an initially spherical wave packet whose maximum moves along the $x$ axis. This means, with $\Delta p_{y}=\Delta p_{z} \equiv \Delta p$ and $p_{y m}=p_{z m}=z_{m}=$ $y_{m}=0$, the calculation simplifies considerably. Although the motion of the maximum will be one-dimensional, this does not hold for the members of the classical ensemble. Simply speaking, the major part of the wave function does not move on this axis. Further, the probability density will be calculated for full periods only. The advantage of this restriction is that the coefficient $g_{5}$ becomes zero and $g_{3}$ and $g_{4}$ are equal. Both is seen if the equation of motion in the $y$ direction (10) is partially integrated:

$$
\begin{aligned}
y(t)= & \left(\int_{0}^{t} \dot{z}\left(t^{\prime}\right) d t^{\prime}+z_{0}\right) \arcsin \left[\frac{R}{\sqrt{1+R^{2}}} \cos (\omega t)\right] \\
& -z_{0} \arcsin \left[\frac{R}{\sqrt{1+R^{2}}}\right] \\
& -\int_{0}^{t} d t^{\prime} \dot{z}\left(t^{\prime}\right) \arcsin \left[\frac{R}{\sqrt{1+R^{2}}} \cos \left(\omega t^{\prime}\right)\right] \\
& +\int_{0}^{t} d t^{\prime} \frac{p_{y}}{\sqrt{1+R^{2} \sin ^{2}\left(\omega t^{\prime}\right)}} .
\end{aligned}
$$

For full periods, the terms depending on $z_{0}$ cancel. The equality of $g_{3}$ and $g_{4}$ is seen by formally integrating Eq. (11) and by comparing the terms proportional to $p_{y}$ to the terms of Eq. (61) proportional to $p_{z}$.

After some algebra, the final result can be written as

$$
\rho(y, z, t) \propto \exp -\frac{\Delta p^{-2}\left(y^{2}+z^{2}\right)+\Delta p^{2}\left[\left(g_{3} z-g_{2} y\right)^{2}+\left(g_{3} y-g_{1} z\right)^{2}\right]}{\left[\Delta p^{-2}+\Delta p^{2}\left(g_{3}^{2}-g_{1} g_{2}\right)\right]^{2}+\left(g_{1}+g_{2}\right)^{2}} .
$$

The factor which renders this proportionality an equality can be found by normalizing the probability density. This will be accomplished once the widths of the wave packet are evaluated.

The next step is to employ the expression for the probability density to derive expressions for the wave packet widths in the relativistic regime. Therefore the quadratic form in the numerator of Eq. (62) needs to be diagonalized such that the mixed terms $y z$ vanish. With the corresponding coordinates $\tilde{Y}, \tilde{Z}$ the normalized probability density adopts the form

$$
\rho(\tilde{Y}, \tilde{Z})=\frac{1}{\pi \cdot \Delta w_{1} \cdot \Delta w_{2}} \exp \left[-\left(\frac{\tilde{Y}}{\Delta w_{1}}\right)^{2}-\left(\frac{\tilde{Z}}{\Delta w_{2}}\right)^{2}\right] .
$$

The transformation from the old to the new coordinates is not important here. It is sufficient to determine the widths $\Delta w_{1,2}$ by means of the eigenvalues. The widths are found to be

$$
\Delta w_{1,2}^{2}=\frac{\left[\Delta p^{-2}+\Delta p^{2}\left(g_{3}^{2}-g_{1} g_{2}\right)\right]^{2}+\left(g_{1}+g_{2}\right)^{2}}{\Delta p^{-2}+\Delta p^{2}\left[g_{3}^{2}+\frac{1}{2}\left(g_{1}^{2}+g_{2}^{2}\right) \pm \frac{1}{2} \sqrt{\left(g_{1}^{2}-g_{2}^{2}\right)^{2}+4 g_{3}^{2}\left(g_{1}+g_{2}\right)^{2}}\right]} .
$$


There is an important result which can be deduced from this complicated looking result. In the relativistic limit of the classical equations of motion (34), (39), and (42), one finds that all of the coefficients $g_{1}, g_{2}, g_{3}$ are proportional to $1 / R$ after $n$ full periods:

$$
\begin{aligned}
g_{1} & =\frac{n}{R \omega}\left[4\left(1+\frac{\pi^{2}}{2}\right) \log 4 R-16.83+\frac{2 \pi}{R}\right] \\
g_{2} & =\frac{4 n \log 4 R}{R \omega} \\
g_{3} & =\frac{2 \pi n \log 4 R}{R \omega} .
\end{aligned}
$$

This means that the greater $R$ is chosen, the smaller the widths will be after $n$ full periods. This behavior has a simple explanation. As the particle approaches the speed of light, time dilation inhibits wave packet spreading. The theoretical limit for the minimal widths is given by the initial widths of $\Delta w_{1,2}=\Delta p^{-1}$. As opposed to the case of linear laser polarization, the dynamics is not unstable in the $z$ direction.

A comparison of the relativistic and the nonrelativistic regime is shown in Fig. 5. The probability densities are plotted according to Eqs. (10), (11), and (62). It is seen that wave packet spreading is reduced in the relativistic case.

\section{B. Linear polarization}

An analogous derivation can be carried out for the case of linear laser polarization. However, the structure of the equations of motion is easier since the equations decouple. Therefore, the solution for each direction can be written in the one-dimensional form:

$$
x(t)=a(t) \cdot p_{0}+b(t) \cdot x_{0}+d(t)
$$

where the functions $a(t), b(t), d(t)$ do not depend on the initial conditions $p_{0}$ and $x_{0}$. As in the case of circular polarization, this needs to be solved for the initial momentum $p_{0}$, inserted into the initial momentum distribution [the one-dimensional analog of Eq. (59)] and finally the initial position has to be averaged [compare Eq. (60)]. Then the one-dimensional probability distribution is found to be

$$
\rho(x, t)=\kappa \exp \left[-\frac{\left[x-b(t) x_{m}-d(t)-a(t) p_{m}\right]^{2}}{\Delta p^{-2} b(t)^{2}+\Delta p^{2} a(t)^{2}}\right]
$$

where $\kappa$ can be adapted to normalize the distribution.

For example, this result can be employed to construct the probability distribution for the $y$ - $z$ plane after a quarter of a laser period for the relativistic case. The coefficients $a(t)$, $b(t), d(t)$ follow from the classical solutions given by Eqs. (15b) and (26):

$$
\rho(y, z)=\tilde{\kappa} \exp \left[-\frac{y^{2}}{\Delta p^{-2}+\Delta p_{z}^{2}(\log 4 R)^{2} /(R \omega)^{2}}\right] \exp \left[-\frac{z^{2}}{3.28 \Delta p^{-2}+\Delta p^{2}(1.53+1.79 \log R)^{2} /(R \omega)^{2}}\right]
$$
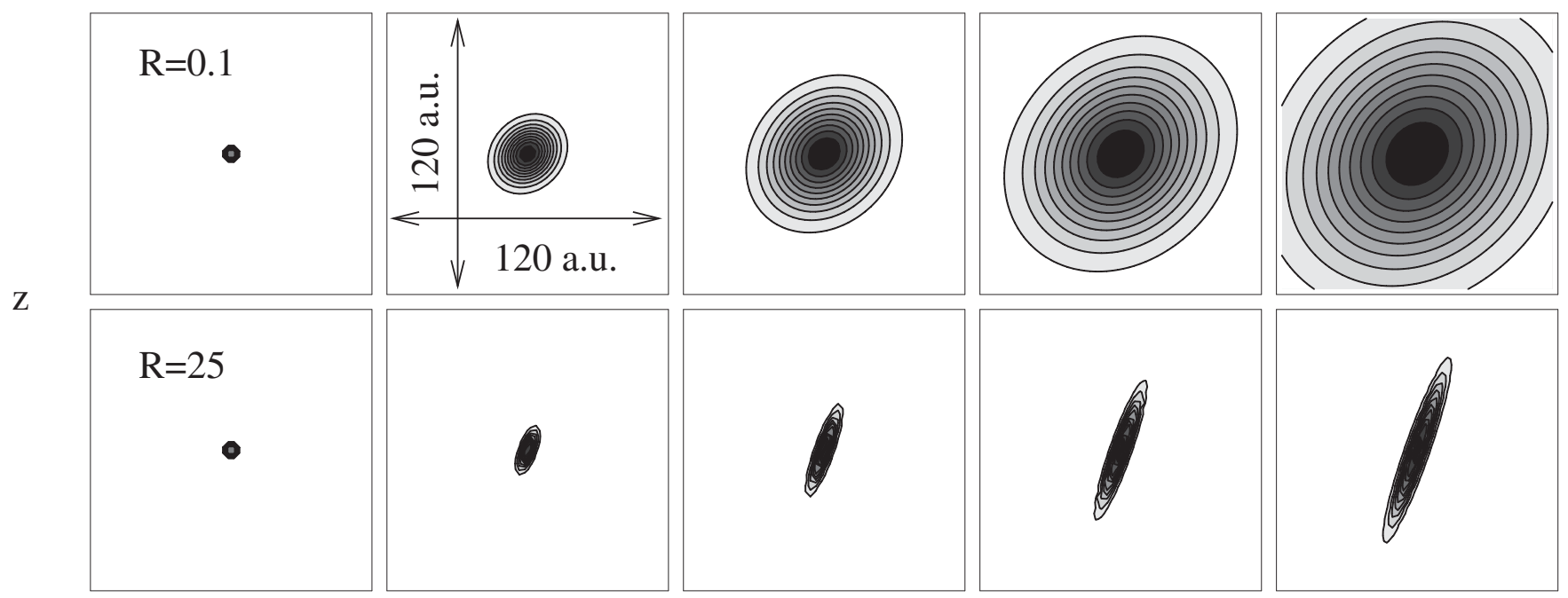

$\mathrm{y}$

FIG. 5. The wave packets in the $y$-z plane, i.e., perpendicular to the electric field, are depicted for counterpropagating circularly polarized laser fields at the initial time and at four consecutive full periods. The initial widths are $\Delta p=0.2$ a.u. In the highly relativistic regime (bottom row, $R=25$ ), wave packet spreading is strongly suppressed compared to the nonrelativistic case (top row, $R=0.1)$. 


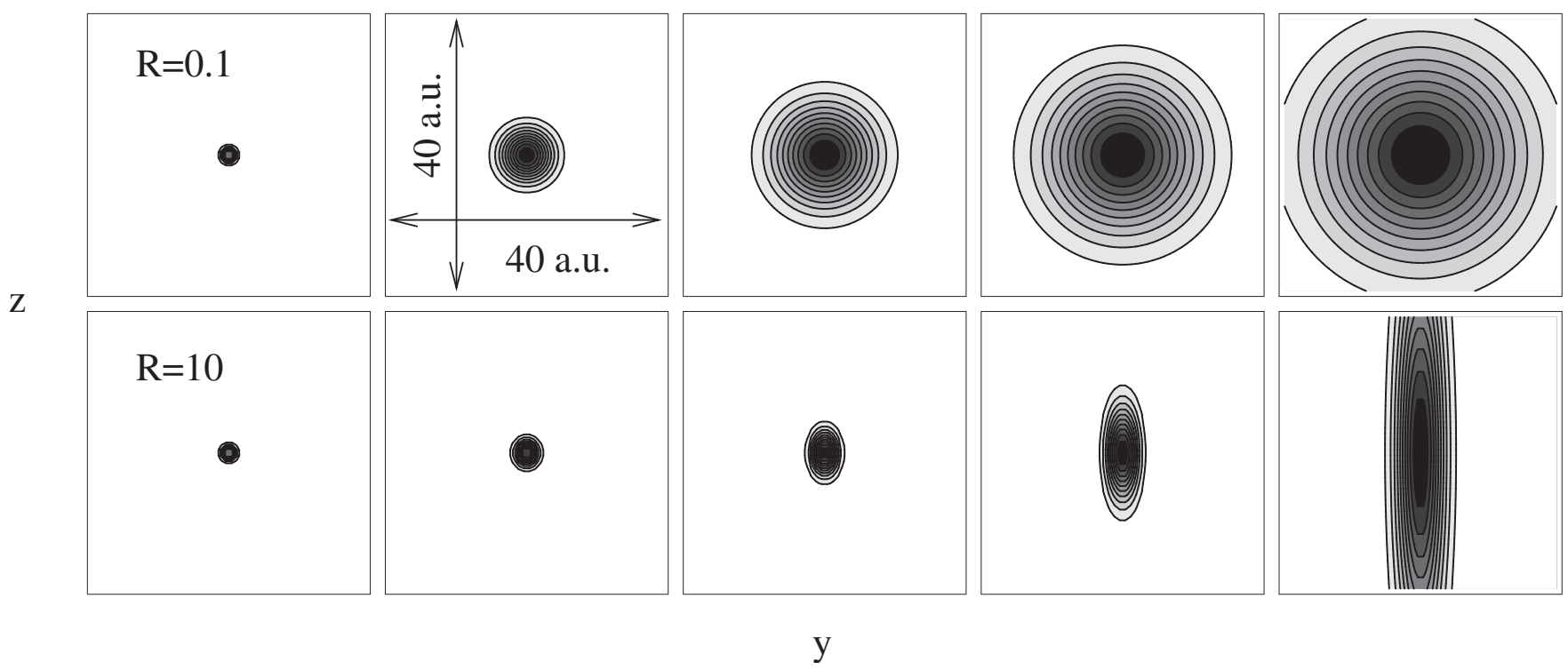

FIG. 6. The nonrelativistic wave packet evolution (top row, $R=0.1$ ) is compared to the relativistic case (bottom row, $R=10$ ) for linearly polarized crossed laser fields. The probability densities in the $y$ - $z$ plane, i.e., perpendicular to the electric field, are shown for the times $\omega t=\{0, \pi / 4, \pi / 2,3 \pi / 4, \pi\}$ (from the left to the right) with the initial widths $\Delta p=0.5$ a.u. As long as the wave packet is small, spreading is suppressed in the relativistic case, but after some time the instability in the $z$ direction becomes dominant.

$\tilde{\kappa}$ is the normalization constant.

Figure 6 depicts the evolution of the wave packet in the $y-z$ plane for a full period, based on Eqs. (8) and (68). Two different parameters $R$ have been chosen to compare the nonrelativistic to the relativistic wave packet dynamics.

It can be seen that wave packet spreading is inhibited in the relativistic case. However, for long times the instability becomes dominant stretching the wave packet in the $z$ direction.

\section{CONCLUSIONS}

Throughout the article it was assumed that the electron in both configurations - linear and circular polarization oscillates close to an ideal axis where the Lorentz force vanishes. For the case the electron can be described nonrelativistically, i.e., the velocity is small compared to the speed of light and terms of higher order than $1 / c$ are negligible, the systems have been described classically and quantum mechanically. It was found that the maximum of a Gaussian wave packet moves like a classical particle. For both configurations, wave packet spreading was found to be similar to that of a free particle.

The strongly relativistic electron dynamics was described analytically by passing over to a classical method where wave packets are described by an ensemble of classical trajectories. This Monte Carlo approach of phase-space averaging requires the knowledge of the classical particle dynamics. Considering a classical electron after a quarter of a period, i.e., at the time when it reaches its maximum velocity for the first time, both configurations give similar results. However, if the time is chosen to be longer, the configuration with linear laser polarization becomes highly unstable in the laser propagation direction, i.e., the distance from the ideal axis increases quickly. This behavior transfers to the wave packet dynamics in the Monte Carlo approach. After a quarter of a laser period when the electron has reached its maximal energy both configurations work equally well, whereas higher laser intensities reduce wave packet spreading. However, for longer times, the case of circular polarization turns out to be much more effective. The reduction of wave packet spreading increases with the strength of the driving laser fields. The configuration of linear laser polarization shows a different behavior. For strong fields, the instability in the laser propagation direction dominates the positive effect of time dilation on spreading, i.e., the wave packet is stretched. This effect increases with stronger laser fields.

In all, it has been found that the dynamics of electrons oscillating close to an axis of vanishing Lorentz force have similar properties in both configurations if the dynamics is nonrelativistic. For short times like a quarter of a laser period, this even holds for the highly relativistic case. Differences occur in the relativistic long-term behavior. As opposed to the case of circular polarization, the configuration with linear polarization shows an instability in the directions of laser propagation.

\section{ACKNOWLEDGMENTS}

Funding by Deutsche Forschungsgemeinschaft, Grant No. KE 721/1-1, is gratefully acknowledged. 
[1] A. L'Huillier, K. J. Schäfer, and K. C. Kulander, J. Phys. B 24, 3315 (1991).

[2] D. B. Milosevic and F. Ehlotzky, Adv. At. Mol. Opt. Phys. 49, 373 (2003).

[3] C. J. Joachain, M. Dörr, and N. Kylstra, Adv. At. Mol. Opt. Phys. 42, 225 (2000).

[4] M. Protopapas, C. H. Keitel, and P. Knight, Rep. Prog. Phys. 60, 389 (1997).

[5] W. Becker et al., Adv. At. Mol. Opt. Phys. 48, 35 (2002).

[6] Y. I. Salamin et al., Phys. Rep. 427, 41 (2006).

[7] F. Ehlotzky, A. Jaron, and J. Z. Kaminski, Phys. Rep. 297, 63 (1998).

[8] F. H.M. Faisal and A. Becker, Laser Phys. 7, 684 (1997).

[9] P. M. Paul et al., Science 292, 1689 (2001).

[10] M. Hentschel et al., Nature (London) 414, 509 (2001).

[11] C. Spielmann et al., Science 278, 661 (1997).

[12] Z. Chang et al., Phys. Rev. Lett. 79, 2967 (1997).

[13] H. Niikura et al., Nature (London) 421, 826 (2003).

[14] M. Lein, Phys. Rev. Lett. 94, 053004 (2005).

[15] S. Baker et al., Science 312, 424 (2006).

[16] J. Itatani et al., Nature (London) 432, 867 (2004).

[17] M. Verschl and C. H. Keitel, Laser Phys. 15, 529 (2005).

[18] G. A. Mourou, C. P. J. Barty, and M. D. Perry, Phys. Today 51, No. 1, 22 (1998).

[19] S. Bahk et al., Opt. Lett. 29, 2837 (2004).

[20] T.E. Cowan et al., Nucl. Instrum. Methods Phys. Res., Sect. A 455, 130 (2000).

[21] K. Nakashima and H. Takabe, Phys. Plasmas 9, 1505 (2002).

[22] S. S. Bulanov, Phys. Rev. E 69, 036408 (2004).

[23] P. Golovinski, M. Dolgopolov, and V. Khlebostroev, J. Nonlinear Opt. Phys. Mater. 5, 139 (1996).

[24] R. Fischer, A. Staudt, and C. H. Keitel, Comput. Phys. Commun. 157, 139 (2004).

[25] G. R. Mocken and C.H. Keitel, J. Phys. B 37, L275 (2004).

[26] C. C. Chirila et al., Phys. Rev. Lett. 93, 243603 (2004).

[27] R. Fischer, M. Lein, and C. H. Keitel, Phys. Rev. Lett. 97, 143901 (2006).

[28] M. Klaiber, K. Z. Hatsagortsyan, and C. H. Keitel, physics/ 0608015.
[29] B. Henrich, K. Z. Hatsagortsyan, and C.H. Keitel, Phys. Rev. Lett. 93, 013601 (2004); K.Z. Hatsagortsyan, C. Müller, and C. H. Keitel, Europhys. Lett. 76, 29 (2006).

[30] N. Kylstra et al., Phys. Rev. Lett. 85, 1835 (2000).

[31] J. de Aldana and L. Roso, J. Opt. Soc. Am. B 19, 1467 (2002).

[32] N. Milosevic, P. B. Corkum, and T. Brabec, Phys. Rev. Lett. 92, 013002 (2004).

[33] Y. I. Salamin, G. R. Mocken, and C. H. Keitel, Phys. Rev. E 67, 016501 (2003).

[34] X. M. Tong and S. I. Chu, Phys. Rev. A 58, R2656 (1998).

[35] J. T. Mendonca, Phys. Rev. A 28, 3592 (1983).

[36] Z.-M. Sheng et al., Phys. Rev. Lett. 88, 055004 (2002).

[37] H. Schwoerer et al., Phys. Rev. Lett. 96, 014802 (2006).

[38] http://www.clf.rl.ac.uk/Facilities/AstraWeb/ AstraGeminiHome.htm

[39] C. Cohen-Tannoudji, B. Diu, and F. Laloë, Quantum Mechanics (Wiley, New York, 1996), Vol. 1.

[40] G. R. Mocken and C. H. Keitel, J. Comput. Phys. 199, 558 (2004).

[41] Y. I. Salamin, G. R. Mocken, and C. H. Keitel, Phys. Rev. ST Accel. Beams 5, 101301 (2002).

[42] N. J. Kylstra, A. M. Ermolaev, and C. J. Joachain, J. Phys. B 30, L449 (1997).

[43] J. W. Braun, Q. Su, and R. Grobe, Phys. Rev. A 59, 604 (1999).

[44] Q. Su, B. A. Smetanko, and R. Grobe, Opt. Express 2, 277 (1998).

[45] U. W. Rathe et al., J. Phys. B 30, L531 (1997).

[46] U.W. Rathe, P. Sanders, and P.L. Knight, Parallel Computing 25, 525 (1999).

[47] J. San Roman, L. Roso, and H. Reiss, J. Phys. B 33, 1869 (2000).

[48] S.-I. Chu and R. Y. Yin, J. Opt. Soc. Am. B 4, 720 (1987).

[49] G. A. Kyrala, J. Opt. Soc. Am. B 4, 731 (1987).

[50] M. Gajda et al., Phys. Rev. A 46, 1638 (1992).

[51] C. H. Keitel, J. Phys. B 29, L873 (1996).

[52] H. Schmitz, K. Boucke, and H.-J. Kull, Phys. Rev. A 57, 467 (1998).

[53] M. Walser, C. Szymanowski, and C. H. Keitel, Europhys. Lett. 48, 533 (1999). 